\title{
SpaRC: Scalable Sequence Clustering using Apache Spark
}

\author{
Lizhen $\mathrm{Shi}^{1}$, Xiandong Meng ${ }^{2,3}$, Elizabeth Tseng ${ }^{4}$, Michael Mascagni ${ }^{1}$, Zhong \\ Wang $^{2,3,5^{*}}$, \\ 1 School of Computer Science, Florida State University, Tallahassee, FL 32304, USA \\ 2 Department of Energy Joint Genome Institute, Walnut Creek, CA 94598, USA \\ 3 Environmental Genomics and Systems Biology Division, Lawrence Berkeley National \\ Laboratory, Berkeley, CA 94720, USA \\ 4 Pacific Biosciences Inc., Menlo Park, CA, 94025, USA \\ 5 School of Natural Sciences, University of California at Merced, Merced, CA, 95343, \\ USA \\ * zhongwang@lbl.gov
}

\begin{abstract}
Whole genome shotgun based next generation transcriptomics and metagenomics studies often generate 100 to 1000 gigabytes (GB) sequence data derived from tens of thousands of different genes or microbial species. De novo assembling these data requires an ideal solution that both scales with data size and optimizes for individual gene or genomes. Here we developed a Apache Spark-based scalable sequence clustering application, SparkReadClust (SpaRC), that partitions the reads based on their molecule of origin to enable downstream assembly optimization. SpaRC produces high clustering performance on transcriptomics and metagenomics test datasets from both short read and long read sequencing technologies. It achieved a near linear scalability with respect to input data size and number of compute nodes. SpaRC can run on different cloud computing environments without modifications while delivering similar performance. In summary, our results suggest SpaRC provides a scalable solution for clustering billions of reads from the next-generation sequencing experiments, and Apache Spark represents a cost-effective solution with rapid development/deployment cycles for similar large scale sequence data analysis problems. The software is available under the Apache 2.0 license at https://bitbucket.org/LizhenShi/sparc
\end{abstract}

\section{Introduction}

Whole genome shotgun sequencing (WGS) using next-generation sequencing technologies followed by de novo assembly is a powerful tool for de novo sequencing large eukaryote transcriptomes (reviewed in 22]) and complex microbial community metagenomes (reviewed in [37]) without reference genomes. Because of the stochastic sampling nature associated with WGS and the presence of sequencing errors, it is necessary for the reads to cover a single gene or genome many times (coverage), typically $30 \mathrm{x}-50 \mathrm{x}$, to ensure high quality de novo assembly [2]. Unlike in single genome sequencing projects where the majority of the genomic regions are equally represented, in transcriptome and metagenome sequencing projects, different species of transcripts or genomes may have very unequal representation, up to several orders of magnitude in abundance difference 16, 23. To obtain a good assembly covering low abundant species 
one would sequence the population at much higher depth than single genome projects. As in practice it is difficult to precisely estimate the required sequencing depth without knowing the community structure, sequencing large transcriptomes and complex metagenomes often generates as much data as the budget allows, producing 100-1000 GB of sequence data or more [15] 33. The largest project so far is the Tara Ocean Metagenomics project where 7.2 Tera bases $(\mathrm{Tb})$ was generated 35].

Since current NGS technologies are not able to read the entire sequence of a genome at once, genomes are broken into small DNA/RNA fragments followed by massive parallel high-throughput sequencing. Different technologies produce sequence reads that vary in length. For example, Illumina technology 17 typically generates about 150 base pair(bp) per read, comparing to $100 \mathrm{bp}$ to 15,000 bp by Pacific Biosciences 28. Assembling these reads to obtain genomes is challenging due to it is both a compute and memory-intensive problem, and this challenge grows exponentially with the size of dataset. Further, the de novo assembly problem is compounded by the quality of sequencing data and the presence of certain genetic complexity (repeat elements, species homology, etc). For a comprehensive review of de novo assembly algorithms please refer to 25. Assembling a large dataset as a whole also requires efficient computing, and these assemblers use either multiple processes on a shared memory architecture (MetaSPAdes 26], MEGAHIT[19], etc) or MPI to distributed on a cluster[11]. The shared memory approach is very hard to scale up to exponentially increased NGS data size. In addition, these assemblers try to tackle the problem as a whole and is not able to produce optimized results as different transcripts or genomes may need individualized optimal parameter settings.

Our work was initially inspired by a "divide-and-conquer" approach presented by DIME [13. DIME first clusters reads based on their overlap, then assembles them separately. It was implemented using Apache Hadoop [4] platform and in theory should scale to large data sets. In practice, however, Hadoop-based implementation has very poor computing efficiency, making it expensive to run on commercial cloud providers. Further, Hadoop-based solutions often produce much larger intermediate files than the input files during the assembly, making them harder to scale to very large datasets.

Recently Apache Spark [5] has overtaken Hadoop in the big data ecosystem due to its fast in-memory computation. Spark has been successfully applied to several genomics problems such as $39,18,7,24,8]$. In this paper we developed a new algorithm based on Spark's GraphX library, called Spark Read Clustering (SpaRC), for parallel construction and subsequent partition of NGS sequence reads. For scalability and computing efficiency SpaRC implemented several heuristics: 1) the pairwise similarity between sequences (edge weight) is estimated by the number of shared k-mers; 2) To control the explosion of edges as data complexity grows, SpaRC sets a max number of edges a vertex can have for each shared k-mer; 3) it adopts an overlapping community detection algorithm - Label Propagation Algorithm (LPA) 30 - to partition the read graph, therefore avoiding the formation of very large partitions due to repetitive or other shared genetic elements between species. We report clustering accuracy and computing performance on both transcriptomic and metagenomic datasets from both short read (Illumina) and long read (PacBio) sequencing platforms.

\section{Methods}

\section{Algorithm Overview}

SpaRC is a generic sequence read clustering algorithm as it is designed for clustering both short and long reads. It first computes the number of shared k-mers between a pair of reads to approximate their overlap, and then builds an undirected read graph followed 
by graph partitioning to achieve read clustering. Specifically, it contains four modules: K-mer Mapping Reads, Graph Construction and Edge Reduction, Graph Partition, and Sequence Retrieval. We describe each of these modules in details as the following.

\section{K-mer Mapping Reads (KMR)}

Given a set of sequence reads, KMR splits them into k-mers according to a predefined k-mer length and only keeps distinct k-mers for each read to avoid low-complexity sequences. KMR keeps track of each k-mer and the reads containing it. The length of $\mathrm{k}$-mer $(k)$ is a parameter to control the sensitivity and specificity of read overlap detection. Shorter k-mers result in more sensitivity but less specificity and vice versa. The ideal k-mer size may depend on sequencing platform, read depth, and sequence complexity.

Generally k-mers appear in only a single read are derived from either sequencing errors or rare molecules, and they take up a large fraction of the total k-mers but are not useful for computing read overlap, therefore they are filtered out. KMR allows users to specify customized filtering criteria (min_kmer_count and max_kmer_count) for more stringency.

\section{Graph Construction and Edge Reduction (GCER)}

GCER constructs a read graph where a node is a read and an edge links two nodes if they share k-mers. Some nodes, if derived from repetitive elements, homologous genes among species or contamination, can have extremely high number of edges (degrees). GCER sets the maximum degree of any vertex for each shared k-mer in a graph (max_degree) as a parameter to reduce unnecessary computation.

After all the vertices and edges are generated, GCER then merges the edges having the same source and destination and filters out those edges with the number of shared k-mers less than the specified parameter min_shared_kmers.

\section{Graph Partition}

SpaRC provides two algorithms for iterative graph partition, Label Propagation Algorithm (LPA) 30] (by default) and Connected Components (CC) [9]. As repetitive elements and homologous genetic elements shared between different molecules/genomes create "overlap communities", in practice LPA in general works much better than CC because LPA allows the resolution of overlap communities. For dataset with very low sequencing coverage CC may be useful.

\section{Sequence Retrieval (AddSeq)}

In the above modules reads are represented by numeric IDs to save memory and storage. Once the clusters are formed, AddSeq retrieves the sequences and get them ready for downstream parallel assembly with a choice of an assembler.

\section{Algorithms}

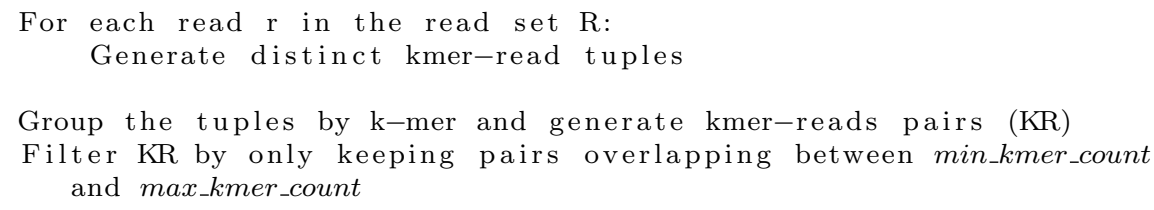




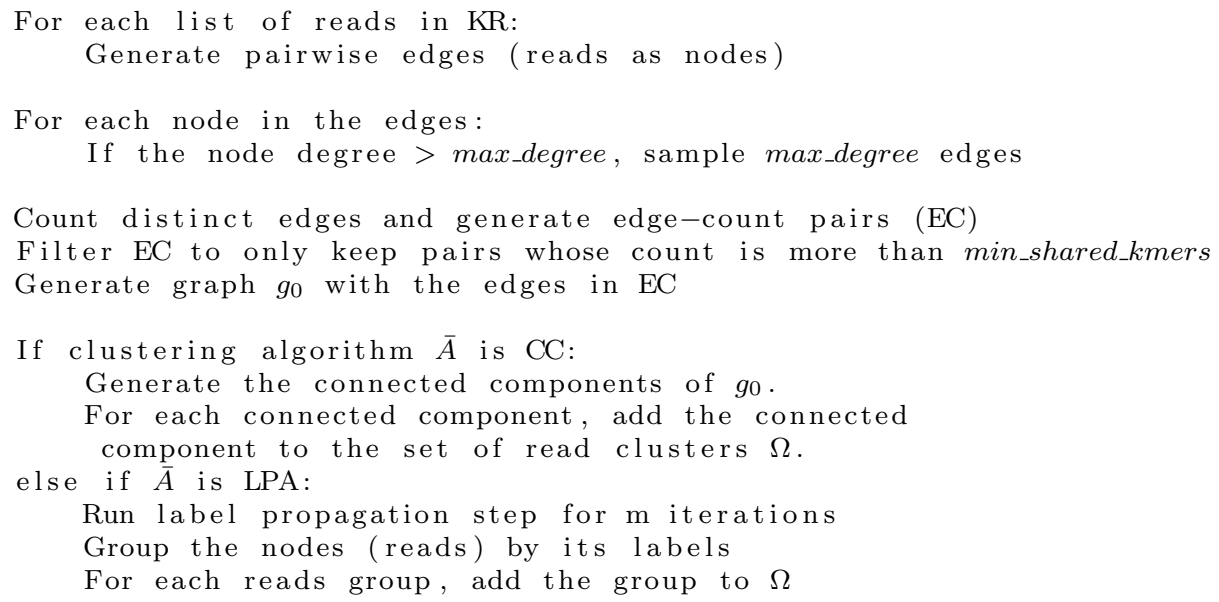

\section{Hardware and Software Environment}

We implement the above algorithm using the Scala (Scala 2.11.8) functional programming language [36. Performance was tested on two closely matched cloud environments, 20 nodes Open Telekom Cloud (OTC) and Amazon's Elastic MapReduce (EMR, emr-5.9.0). For these two clusters, one node is used as the master and all other nodes are used as workers. Configuration details are shown in Table 1.

Table 1. Configuration for OTC and AWS EMR

\begin{tabular}{|l|l|l|}
\hline & OTC & AWS EMR \\
\hline \# of cores/node & 8 & 8 \\
\hline Memory/node & 64 & 61 \\
\hline Storage/node & $500 \mathrm{~GB}$ SSD & $160 \mathrm{~GB}$ SSD \\
\hline Ethernet & $1 \mathrm{Gbps}$ & $10 \mathrm{Gbps}$ \\
\hline Spark version & 2.1 .1 & 2.2 .0 \\
\hline Hadoop version & 2.7 .3 & 2.7 .3 \\
\hline Cluster mode & Standalone & YARN \\
\hline \# of executors/node & 3 & 3 \\
\hline Driver memory & $55 \mathrm{~GB}$ & $40 \mathrm{~GB}$ \\
\hline Driver cores & 5 & 5 \\
\hline Memory/executor & $18 \mathrm{~GB}$ & $16 \mathrm{~GB}$ \\
\hline Cores/executor & 2 & 2 \\
\hline HDFS Block Size & $32 \mathrm{MB}$ & $32 \mathrm{MB}$ \\
\hline
\end{tabular}

\section{Datasets}

We tested the performance of SpaRC on both simulated and real world datasets. A maize sequence dataset we generated previously from [23], and the cow rumen metagenome dataset [14], from which we generated subsets of 1 to 100 (GB) in fastq, for testing scalability. A mock dataset containing 26 genomes is used to verify accuracy. Three long read transcriptome datasets were provided by PacBio. The datasets are described in Table 2 
Table 2. Metrics of test datasets

\begin{tabular}{|l|l|l|l|}
\hline Dataset & \# Species & Read Length (bp) & Size(GB) \\
\hline $\begin{array}{l}\text { PacBio1 (Human Alzheimer } \\
\text { brain transcriptome) }\end{array}$ & High & $300-30816$ & 3.8 \\
\hline $\begin{array}{l}\text { PacBio2 (Human UHRR }+ \\
\text { synthetic RNA, 2Cell) }\end{array}$ & High & $62-14621$ & 1 \\
\hline $\begin{array}{l}\text { PacBio3 (Human UHRR } \\
+ \text { synthetic RNA, 3Cell) }\end{array}$ & High & $54-14833$ & 1.8 \\
\hline Maize transcriptome & High & 151 X2 & 4 \\
\hline Mock metagenome & Low (26) & $(90-150) \times 2$ & 15 \\
\hline Cow Rumen metagenome & Medium & $100 \times 2$ & 100 \\
\hline
\end{tabular}

\section{Results}

\section{SpaRC clustering accuracy}

In order to measure the clustering performance of SpaRC, we used two sets of real world data sets with "known answers" and ran SpaRC to obtain clusters.

The first dataset is derived from human Alzheimer whole brain transcriptome sequenced by PacBio consisting of 1,107,889 full-length transcript sequences. The transcript sequences were first clustered together based on an isoform-level clustering algorithm [12], then the consensus sequence from each cluster were mapped back to the human genome to identify which loci it came from. Reads coming from clusters where the mapped genomic location overlap by at least $1 \mathrm{bp}$ are considered to be from the same loci. This is the theoretical limit for overlap-based clustering algorithms. The second data set is two million Illumina short metagenome reads (150bp) sampled from a mock microbial community consisting of 26 genomes described previously 34. Clusters are defined similarly as above for the PacBio transcriptome data set.

By comparing the SpaRC clusters to "known answers" in the above two datasets, we measured SpaRC's performance by cluster purity, and cluster completeness. Here cluster purity is defined as the percentage of reads belonging to the dominant known cluster for each SpaRC cluster, and completeness is defined as the maximum percentage of reads from a known cluster could be captured by a SpaRC cluster. It is worth noting that cluster completeness will be an underestimation of the true cluster completeness as the "known answers" are overestimation as described above. As the sensitivity of overlap detection is heavily influenced by the read length, in the Illumina metagenome dataset we joined the reads in a pair that are pair-end sequenced to double the read length for clustering.

In both experiments SpaRC clustered the majority of the reads (PacBio: $82.65 \%$, Ilumina: 98.3\%), and generated very pure clusters ( Fig 1 A,E). For the impure read clusters in the both datasets, the contamination events seem to be relative low, as their purity increases with cluster size (Fig 1 B,$F)$.

Clustering long reads achieved a much higher completeness than short reads, with many more clusters that have completeness $\geq 90 \%(84.88 \%, \mathrm{n}=9,578, \mathrm{Fig} 1 \mathrm{C})$, comparing to short read clusters $(37.19 \%, n=37,879)$, Fig 1 G). For the long read transcriptome dataset, the completeness improves as cluster size is getting bigger, suggesting more copies of a transcript increases the chance of finding overlap. For the short read metagenome dataset, larger clusters tend to have lower completeness. As the copy number of each genome is a constant and larger clusters translate to larger genome regions, they are more prone to be broken into smaller clusters due to loss of some overlaps.

We also tested whether or not completeness would get worse if the read pairs in the 
short read dataset were not joined. This indeed is the case, as clusters that have completeness $\geq 90 \%$ is decreased to $6.08 \%$ and more small clusters are produced $(\mathrm{n}=42,181)$.

\section{Accuracy comparison with alternative solutions}

To assess whether using SpaRC improves recovery of the known synthetic spike-in transcripts in the PacBio human data, clustering results from SpaRC were compared with the minimap-based 20] clustering results and run through the PacBio Iso-Seq clustering pipeline [27]. The results (Table 3) from SpaRC show comparable results with slightly improved recovery of the synthetic spike-in transcripts (more true positives) and slightly reduced artifacts (fewer false positives). The difference seems to be more pronounced when sequence depth is lower (PacBio2).

Table 3. Comparison of recovered synthetic SIRV transcripts in the PacBio human transcriptome data.

\begin{tabular}{|c|l|l|l|l|}
\hline \multirow{2}{*}{ Dataset } & \multicolumn{2}{|c|}{ SpaRC } & \multicolumn{2}{l|}{ Minimap } \\
\cline { 2 - 5 } & TP & FP & TP & FP \\
\hline PacBio2 (375k reads) & 57 & 13 & 54 & 17 \\
\hline PacBio3 (623k reads) & 61 & 11 & 61 & 14 \\
\hline
\end{tabular}

\section{Data Complexity has a major effect on SpaRC execution time}

As introduced above, SpaRC consists of 4 steps: KMR, GCER, Graph Partition, and AddSeq. To measure the computing efficiency of each step on data with different complexity (number of species, see Table 2), we run SpaRC against Human Alzheimer transcriptome, Maize transcriptome, and Cow Rumen metagenome with the same data size (about 4GB) on OTC.

We found different datasets give rise to very different execution times (Fig 2). First of all, complex metagenome dataset has many more unique kmers, which requires longer KMR running time. Same sized transcriptome dataset, Pabio has more k-mers than Illumina, presumably due to higher error rate. Second, reads from complex metagenome dataset typically have fewer edges than transcriptome because many species do not have sufficient sequencing coverage. Longer reads tend to have more edges because they have more k-mers (Table 4). Finally, even given comparable number of total edges (Table 4), LPA step takes significantly longer execution time for long read transcriptome dataset than the short read transcriptome dataset. This is because each long read dataset has more edges per vertex than short read, and GraphX's LPA implementation uses vertex-cut for graph partition [38, resulting in more copies of vertices, which in turn translates into higher time cost in each LPA iteration.

Table 4. Metrics of different datasets

\begin{tabular}{|l|l|l|l|l|}
\hline Dataset & \# of k-mers & \# of edges & \# of nodes & Avg degrees per node \\
\hline PacBio1 & $179,039,835$ & $263,116,527$ & $1,027,204$ & 512 \\
\hline Maize & $96,643,966$ & $298,631,852$ & $11,465,314$ & 52 \\
\hline Cow Rumen & $46,027,775$ & $41,155,061$ & $4,001,389$ & 20 \\
\hline
\end{tabular}

Among the steps in the workflow, AddSeq is the simplest step and takes very little time (no more than 1 minute) for all datasets. 
A

C
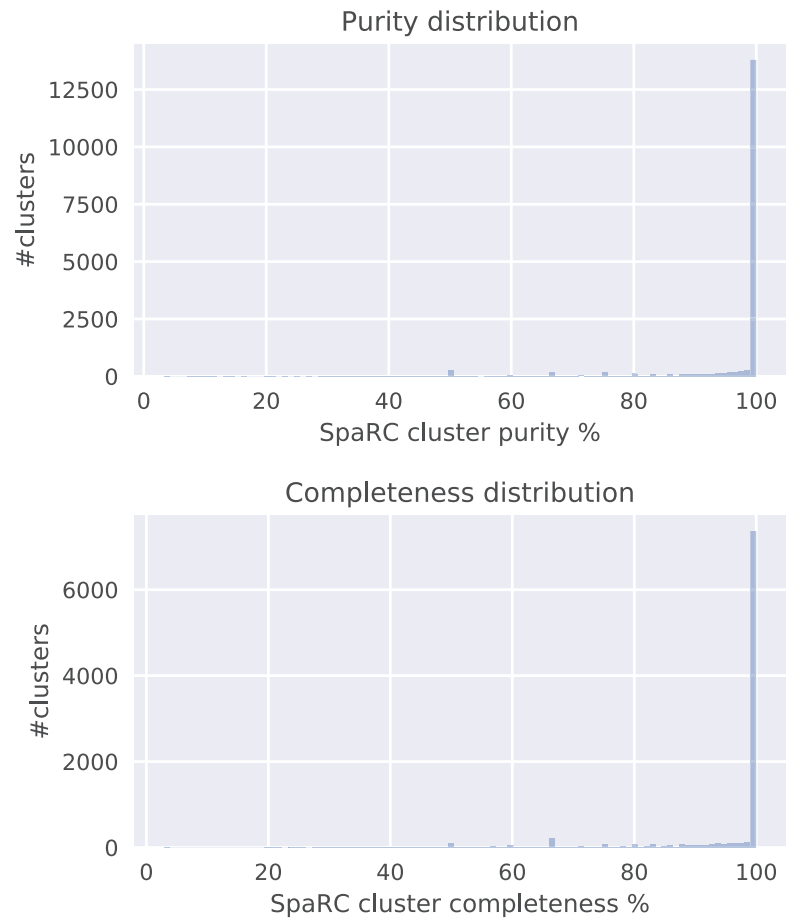

E

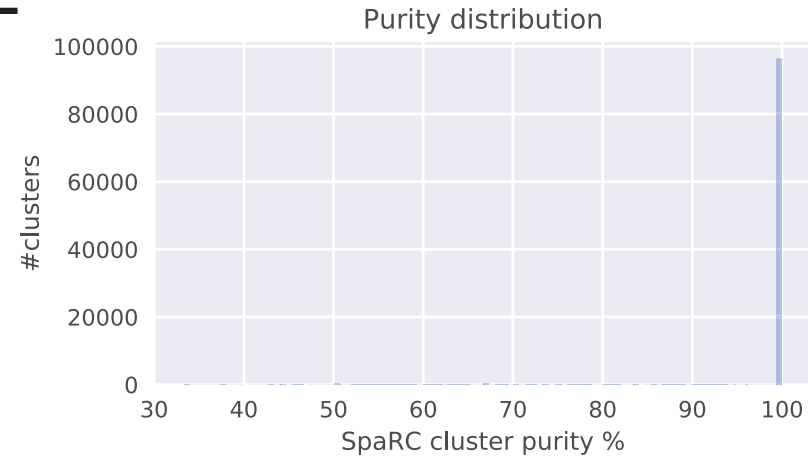

G

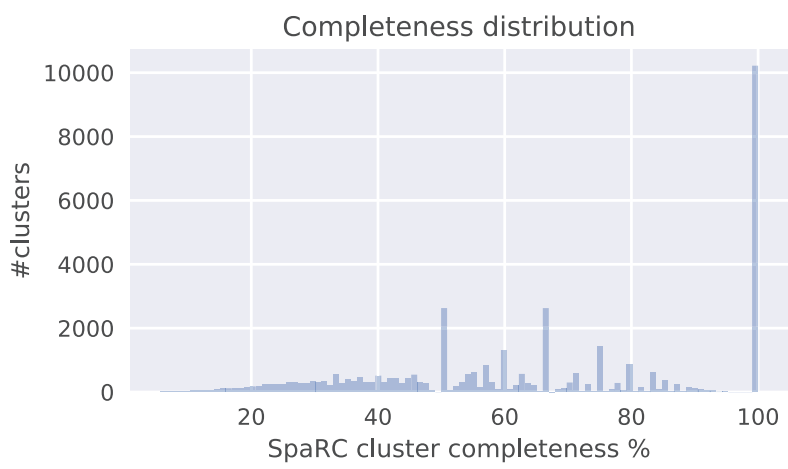

B

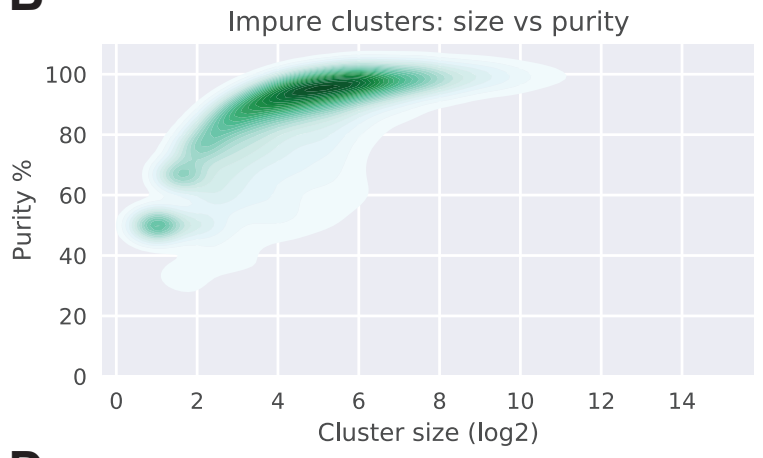

D

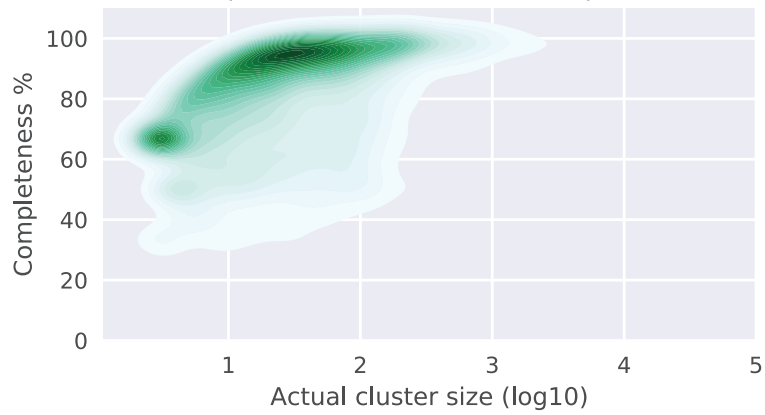

F

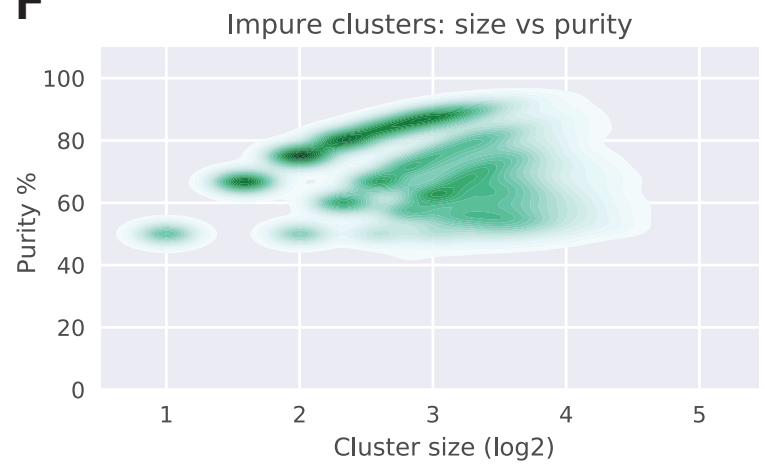

H

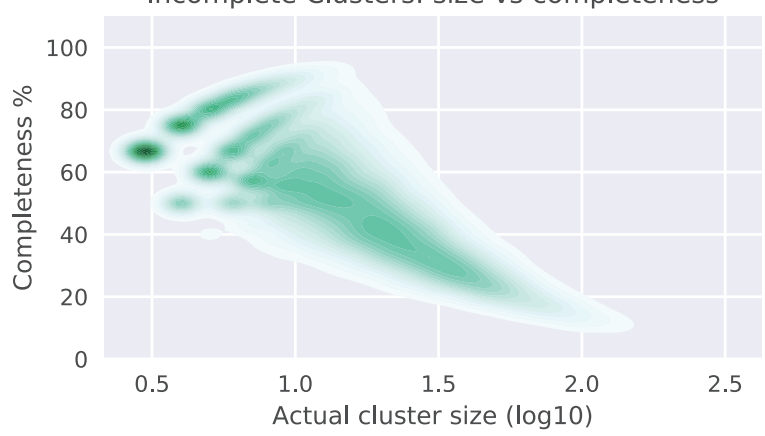

Figure 1. SpaRC's clustering performance on long and short reads. A-D) Pacbio transcriptome and E-H) Illumina metagenome. 


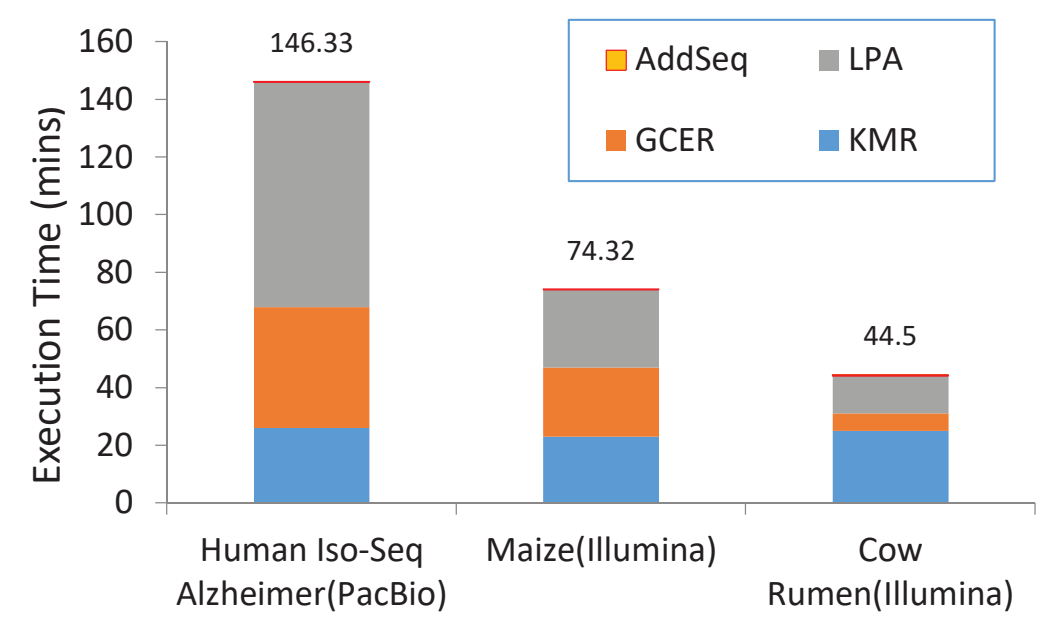

Figure 2. Performance difference between datasets

\section{Degree of Parallelism}

on SpaRC's computing performance

It has been reported parallelism level has a major effect on the performance of the Spark applications [1]. In earlier versions of SpaRC based on Spark version 1.6, we also observed smaller parallelism level led to poor performance due to some jobs take too long to finish due to data imbalance (data not shown). We therefore evaluated the effect of parallelism level on the overall execution time of the current SpaRC software.

Because the size of each data partition is also a function of input data size, we run multiple SpaRC experiments over 20GB and 50GB Cow Rumen dataset on OTC, each with a Spark default parallelism (spark.default.parallelism) value ranging from 50 to 20,000. Once set, Spark automatically sets the number of partitions of an input file according to its size for distributed shuffles.

As shown in Fig 3 , we found the performance of SpaRC does not vary much over several orders of magnitude in parallelism, for both of the two datasets tested. As long as the parallelism is not extreme (less than 100 or over 1 million), SpaRC's performance is quite consistent. When there are too few data partitions, performance suffers because of cluster resource under utilization. In contrast, when there are too many data partitions, there might be excessive overhead in managing small tasks. It is not necessary, at least in this case, to adjust the default parallelisms.

It is worth noting that Spark relies on Hadoop file system (HDFS) which has a default partition size $64 \mathrm{MB}$. Our previous work showed that bioinformatics applications can benefit from setting it to 32MB [32], therefore in SpaRC we recommend setting HDFS default partition size to $32 \mathrm{MB}$.

\section{SpaRC scales near linearly with input data and compute nodes}

We designed two different experiments to measure the scalability of the SpaRC. The first one tests its data scalability as more input are added on a fixed-sized cluster, and the second measures its horizontal scalability as more nodes are added to the cluster to compute the same input. For data scalability test we use 20GB, 40GB, 60GB, 80GB, and 100GB fastq datasets from Cow Rumen metagenome. The sequence retrieval step (AddSeq) is not shown due to its negligible processing time (as mentioned in the above).

We report in Fig 4 the result of the first experiment varying input data size and maintaining the number of nodes in the OTC cluster to a fixed value (20). The KMR 


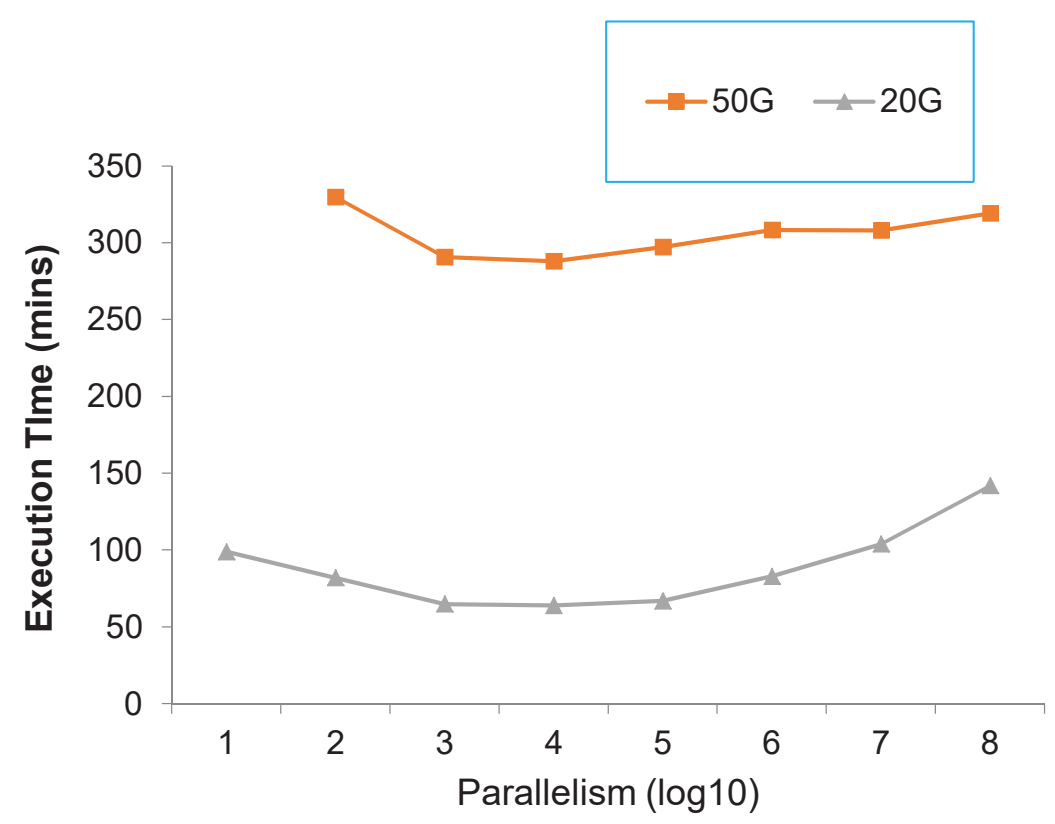

Figure 3. The effect of parallelism level on the total execution time

and GCER Step scale up linearly as expected, while LPA step scales up near linearly, consistent with previously reported $[30$.

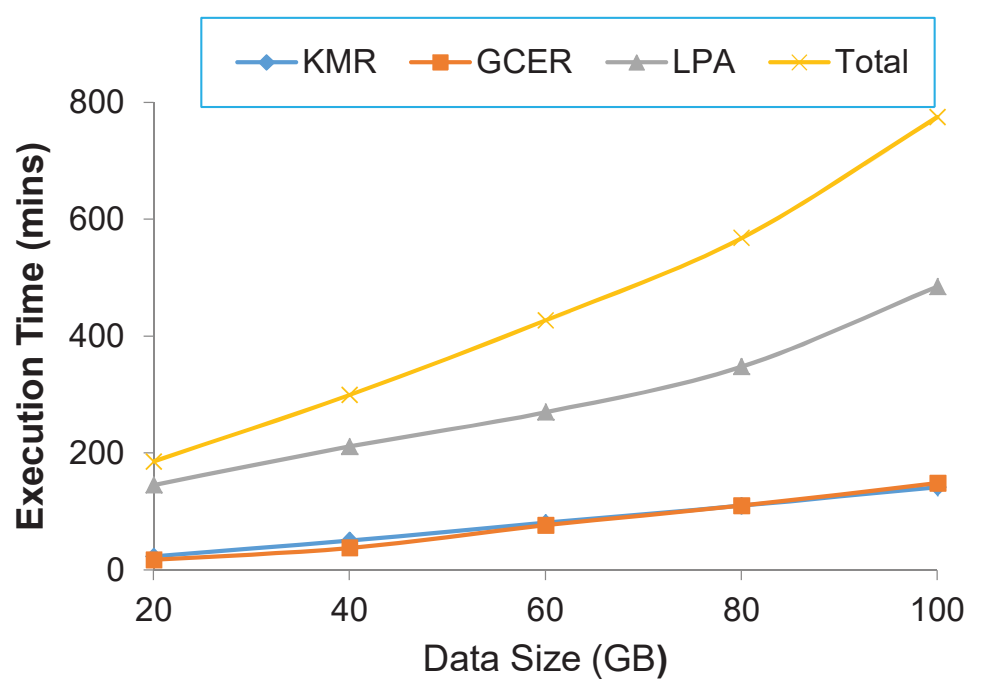

Figure 4. Scalability with different input size

We next tested SpaRC performances by keeping the input size fixed (10GB, 50GB) but varying number of nodes. As shown in Fig 5 , the compute time required for each stage and the total time decreases as the number of nodes increases. However, there appears to be a "sweet spot" for each specific input size (10 nodes for 10GB, 50 for $50 \mathrm{~GB}$, respectively). Before the number of nodes reaches this spot, every doubling in number of nodes translates into approximately halving the compute time. However, the slope of time saving is decreasing when the node number increases beyond the spot. 
This phenomenon can be explained by the Amdahl's law [3] in parallel computing. Overall, we achieve the near-linear scalability as other spark-based tools 8, 31, suggesting SpaRC scales well to the number of nodes.

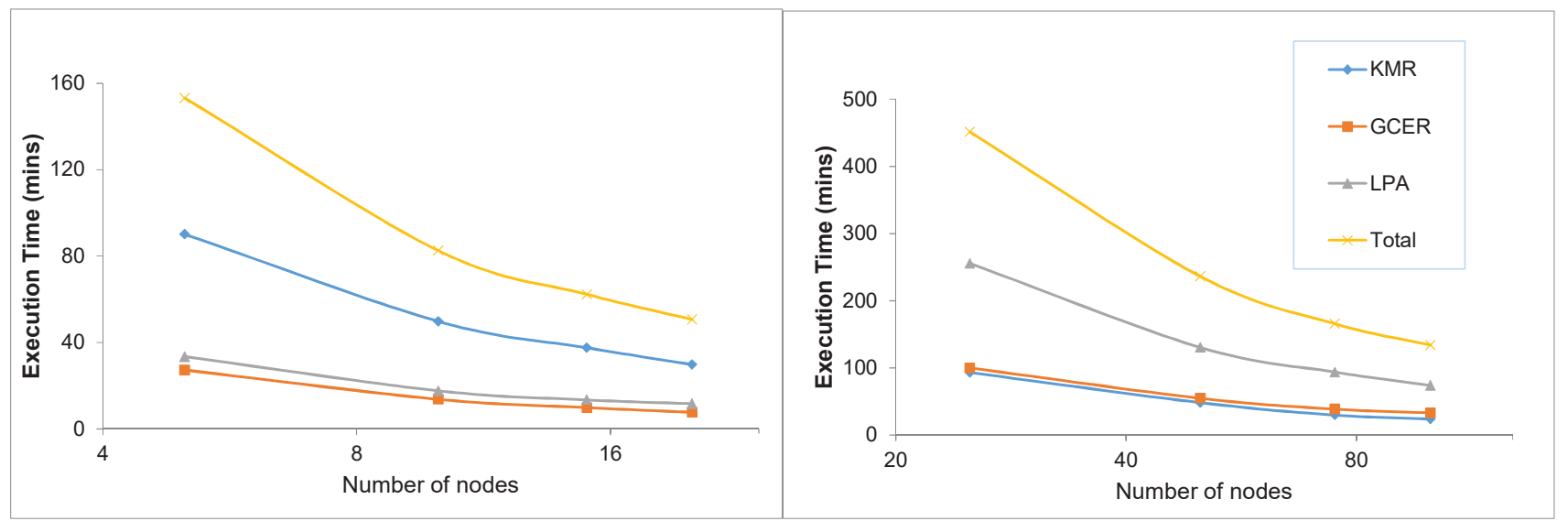

Figure 5. scalability with different number of nodes (Left: 10GB; Right: 50GB)

\section{Conclusion and Discussion}

Metagenome and transcriptome assembly is challenging due to both its scale and complexity. Here we developed a scalable algorithm, SpaRC, for large-scale metagenome and transcriptome reads clustering to enable downstream assembly optimization tailored towards individual gene/genome. SpaRC takes advantage of Apache Spark for scalability, efficiency, fast development and flexible running environments. We evaluated SpaRC on both transcriptome and metagenome datasets and demonstrated that SpaRC produces accurate results comparable to state-of-the-art clustering algorithms.

Since Apache Spark is still a very young project undergoing heavy development, some of its components have not been stable and/or optimized. For example, currently LPA is implemented in GraphX using the pregel interface 21 instead of in GraphFrame [10], which did not take the full advantage of the scalability and efficiency of DataFrame API 6]. Current LPA function in GraphFrame is a simple wrapper of the method in GraphX, and it is neither space nor time efficient. Since it cumulatively caches the results of each iteration for job recovery, disk usage often explodes as the number of iterations increases. Furthermore, if one executor dies, all of its cached data is lost and the whole process has to start from scratch. Creating a checkpoint for each iteration like the GraphFrame version of connect component should alleviate this problem.

We observed the clusters produced tend to be too small when the read length is short (e.g., single-end metagenomic dataset on Illumina platform). For pair-end sequencing datasets one can merge (if they overlap) or concatenate the two ends to increase the cluster size. Decreasing k-mer size, or requiring less shared k-mers should also help increase cluster size. However, this may lead to decrease of purity. One potential solution is to run an additional binning or scaffolding step (using pair-end or long reads if available) after assembling each cluster of reads into contigs, a common step in metagenome assemblies.

Based on our experience running SpaRC on OTC and AWS cloud computing environments give similar performance. We also attempted to run SpaRC on HPC environments, including NERSC's Cori system 
(http://www.nersc.gov/users/computational-systems/cori/) and Pittsburgh Supercomputing Center's Bridge system (https://www.psc.edu/bridges). On these systems, the Hadoop and Spark frameworks are provisioned in an on-demand fashion to allow Spark jobs. Although SpaRC runs well on small datasets on both systems, scaling up to larger dataset $(i 1 \mathrm{~Gb})$ failed because of various job scheduling and network problems. More research is needed to get SpaRC run in similar HPC environments.

\section{Acknowledgments}

We thank Drs XXX for critical reading the manuscript. We thank members of NERSC, especially Dr. Lisa Gerhardt and Mr. Evan Racah for their support to run SpaRC on Cori system. We thank members of Pittsburgh Supercomputing Center (PSC), especially Dr. Philip Blood and Mr. Bryon Gill for their support to run SpaRC on the Bridge system. We thank Dr. Hui Zheng and Mr. Billy Xu from Huawei Inc. for their support to run SpaRC on the OTC system.

\section{Funding}

Xiandong Meng and Zhong Wang's work was supported by the U.S. Department of Energy, Office of Science, Office of Biological and Environmental Research under Contract No. DE-AC02-05CH11231. The OTC computing resource is provided by Huawei Inc. through research collaboration. Part of the Amazon Web Service computational resources was supported by the AWS Cloud Credits for Research Program "EDU_R_RS_FY2015_Q4_DOI-JointGenomeInstitute_Wang". Computing resource on PSC's Bridge system was supported by an XESED grant no "MCB170069".

\section{References}

[1] Anas Abu-Doleh and Ümit V Çatalyürek. "Spaler: Spark and GraphX based de novo genome assembler". In: Big Data (Big Data), 2015 IEEE International Conference on. IEEE. 2015, pp. 1013-1018.

[2] Subramanian S Ajay et al. "Accurate and comprehensive sequencing of personal genomes". In: Genome research 21.9 (2011), pp. 1498-1505.

[3] Amdahl's law. URL: https://en.wikipedia.org/wiki/Amdahl\%27s_law

[4] Apache Hadoop. URL: http://hadoop.apache.org/.

[5] Apache Spark. URL: http://spark.apache.org/

[6] Michael Armbrust et al. "Spark sql: Relational data processing in spark". In: Proceedings of the 2015 ACM SIGMOD International Conference on Management of Data. ACM. 2015, pp. 1383-1394.

[7] Amir Bahmani et al. "SparkScore: leveraging apache spark for distributed genomic inference". In: Parallel and Distributed Processing Symposium Workshops, 2016 IEEE International. IEEE. 2016, pp. 435-442.

[8] Marcelo Rodrigo de Castro et al. "SparkBLAST: scalable BLAST processing using in-memory operations". In: BMC bioinformatics 18.1 (2017), p. 318.

[9] Connected component (graph theory). URL: https://en.wikipedia.org/wiki/Connected_component_(graph_theory). 
[10] Ankur Dave et al. "Graphframes: an integrated api for mixing graph and relational queries". In: Proceedings of the Fourth International Workshop on Graph Data Management Experiences and Systems. ACM. 2016, p. 2.

[11] Evangelos Georganas et al. "HipMer: an extreme-scale de novo genome assembler". In: High Performance Computing, Networking, Storage and Analysis, 2015 SC-International Conference for. IEEE. 2015, pp. 1-11.

[12] Sean P Gordon et al. "Widespread polycistronic transcripts in fungi revealed by single-molecule mRNA sequencing". In: PloS one 10.7 (2015), e0132628.

[13] Xuan Guo et al. "Dime: A novel framework for de novo metagenomic sequence assembly". In: Journal of Computational Biology 22.2 (2015), pp. 159-177.

[14] Matthias Hess et al. "Metagenomic discovery of biomass-degrading genes and genomes from cow rumen". In: Science 331.6016 (2011), pp. 463-467.

[15] Adina Chuang Howe et al. "Tackling soil diversity with the assembly of large, complex metagenomes". In: Proceedings of the National Academy of Sciences 111.13 (2014), pp. 4904-4909.

[16] Jennifer B Hughes et al. "Counting the uncountable: statistical approaches to estimating microbial diversity". In: Applied and environmental microbiology 67.10 (2001), pp. 4399-4406.

[17] Illumina. URL: https://www.illumina.com/.

[18] Max Klein et al. "Biospark: scalable analysis of large numerical datasets from biological simulations and experiments using Hadoop and Spark". In: Bioinformatics 33.2 (2017), pp. 303-305.

[19] Dinghua Li et al. "MEGAHIT: an ultra-fast single-node solution for large and complex metagenomics assembly via succinct de Bruijn graph". In: Bioinformatics 31.10 (2015), pp. 1674-1676.

[20] Heng Li. "Minimap and miniasm: fast mapping and de novo assembly for noisy long sequences". In: Bioinformatics 32.14 (2016), pp. 2103-2110.

[21] Grzegorz Malewicz et al. "Pregel: a system for large-scale graph processing". In: Proceedings of the 2010 ACM SIGMOD International Conference on Management of data. ACM. 2010, pp. 135-146.

[22] Jeffrey A Martin and Zhong Wang. "Next-generation transcriptome assembly". In: Nature Reviews Genetics 12.10 (2011), pp. 671-682.

[23] Jeffrey A Martin et al. "A near complete snapshot of the Zea mays seedling transcriptome revealed from ultra-deep sequencing". In: Scientific reports 4 (2014).

[24] Matt Massie et al. "Adam: Genomics formats and processing patterns for cloud scale computing". In: EECS Department, University of California, Berkeley, Tech. Rep. UCB/EECS-2013-207 (2013).

[25] Jason R Miller, Sergey Koren, and Granger Sutton. "Assembly algorithms for next-generation sequencing data". In: Genomics 95.6 (2010), pp. 315-327.

[26] Sergey Nurk et al. "metaSPAdes: a new versatile metagenomic assembler". In: Genome Research 27.5 (2017), pp. 824-834.

[27] PacBio Iso-Seq clustering pipeline. URL: https://github.com/PacificBiosciences/IsoSeq_SA3nUP.

[28] Pacific Biosciences. URL: http://www.pacb.com/. 
[29] Pavel A Pevzner, Haixu Tang, and Michael S Waterman. "An Eulerian path approach to DNA fragment assembly". In: Proceedings of the National Academy of Sciences 98.17 (2001), pp. 9748-9753.

[30] Usha Nandini Raghavan, Réka Albert, and Soundar Kumara. "Near linear time algorithm to detect community structures in large-scale networks". In: Physical review E 76.3 (2007), p. 036106.

[31] Zeehasham Rasheed and Huzefa Rangwala. "A Map-Reduce framework for clustering metagenomes". In: Parallel and Distributed Processing Symposium Workshops 83 PhD Forum (IPDPSW), 2013 IEEE 27th International. IEEE. 2013, pp. $549-558$.

[32] Lizhen Shi et al. "A case study of tuning MapReduce for efficient Bioinformatics in the cloud". In: Parallel Computing 61 (2017), pp. 83-95.

[33] Weibing Shi et al. "Methane yield phenotypes linked to differential gene expression in the sheep rumen microbiome". In: Genome Research 24.9 (2014), pp. 1517-1525.

[34] Esther Singer et al. "Next generation sequencing data of a defined microbial mock community". In: Scientific data 3 (2016), p. 160081.

[35] Shinichi Sunagawa et al. "Structure and function of the global ocean microbiome". In: Science 348.6237 (2015), p. 1261359.

[36] The Scala Programming Language. URL: https://www.scala-lang.org/.

[37] Susannah Green Tringe and Edward M Rubin. "Metagenomics: DNA sequencing of environmental samples". In: Nature reviews genetics 6.11 (2005), pp. 805-814.

[38] Reynold S Xin et al. "Graphx: A resilient distributed graph system on spark". In: First International Workshop on Graph Data Management Experiences and Systems. ACM. 2013, p. 2.

[39] Xingjian $\mathrm{Xu}$, Zhaohua Ji, and Zhang Zhang. "CloudPhylo: a fast and scalable tool for phylogeny reconstruction". In: Bioinformatics 33.3 (2016), pp. 438-440. 